Original Article

\title{
FORMULATION AND IN VITRO EVALUATION OF POLY-(D, L-LACTIDE-CO-GLYCOLIDE) (PLGA) NANOPARTICLES OF ELLAGIC ACID AND ITS EFFECT ON HUMAN BREAST CANCER, MCF-7 CELL LINE.
}

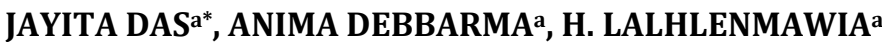

aDepartment of Pharmacy, Regional Institute of Paramedical and Nursing Sciences, Zemabawk, Aizawl, Mizoram 796017, India

Email: jayitadas631@gmail.com

Received: 08 May 2021, Revised and Accepted: 07 Jul 2021

\begin{abstract}
Objective: The aim of this experiment was to develop ellagic acid (EA) loaded poly (D,L-lactide-co-glycolide) (PLGA) nanoparticles for tumourspecific drug delivery. The phytochemical EA is a potential antioxidant, anticarcinogenic and antimutagenic. Due to its low solubility and permeability, it falls under class IV of the BCS classification.

Methods: PLGA nanoparticles were prepared by a method established on the concept of single emulsification-solvent evaporation by using TWEEN ${ }^{\circledR 2} 20$ as a cosolvent for solubilizing the drug. While developing this method, polyvinyl alcohol (PVA), was implemented.

Results: The stabilized formulation was with a particle size of $174.2 \mathrm{~nm}$, which is ideal for tumour accumulation. The SEM images confirmed that the NPs have spherical shape. The resulting NPs were predominantly spherical and of uniform size and shape. Initial release of EA from nanoparticles in pH 7.4 phosphate buffer was quick, followed by a steady sustained release. The in vitro cytotoxicity study using MTT was also performed on the human breast cancer, MCF-7 cell line and EA-NPs were found to successively reduce the IC 50 values which thereby revealed the pronounced cytotoxic effect of the formulation.
\end{abstract}

Conclusion: After performing this experiment, we can conclude that the polymeric nanoparticles are efficient and suitable form of drug delivery of ellagic acid exhibiting potential anti-tumour activity.

Keywords: Ellagic acid nanoparticles, TWEEN ${ }^{\circledR 20}$, Poly vinyl alcohol, MTT assay, Kinetic modelling

(C) 2021 The Authors. Published by Innovare Academic Sciences Pvt Ltd. This is an open access article under the CC BY license (https://creativecommons.org/licenses/by/4.0/) DOI: https://dx.doi.org/10.22159/ijcpr.2021v13i5.1887 Journal homepage: https://innovareacademics.in/journals/index.php/ijcpr

\section{INTRODUCTION}

Nanoparticles are coherent drug delivery systems for enhancing the bioavailability of poorly water-soluble drugs. There are several approaches to enhance drug bioavailability. Among these approaches, nano-encapsulation using PLGA (Polylactic-co-glycolic acid), which is a biocompatible polymeric nanocarrier, is a very favourable one. PLGA is a very well-known biodegradable and biocompatible polymer consented by the US Food and Drug Administration (FDA) and the European Medicine Agency (EMA). It has been used in a variety of biomedical devices and tissue engineering branches. Different therapeutics have been encapsulated in PLGA nano-and microparticles for several purposes such as vaccination or cardiovascular and cancer treatments [1]. PLGA-NPs bind drugs with poor solubility and extravasation through the tumour vasculature by the enhanced permeability and retention effect. The objective of this study was to develop EA-NPs. The nanoparticles were characterized in terms of size by scanning electron microscopy (SEM) and dynamic light scattering (DLS). Drug loading, entrapment efficiency, in vitro release profile and in vitro cytotoxicity assay using MTT on the human breast cancer cell line, MCF-7 [2].

Ellagic acid (EA), a phytochemical which is extensively found in berries, is a potential antioxidant, anticarcinogenic and antimutagenic. Due to its low solubility and permeability, EA falls under class IV of the BCS classification. EA is not only insoluble in water but also is difficult to solubilize in commonly used organic solvents in sufficient quantities for formulation into the nanoparticulate dosage form. The choice of a particular method of encapsulation of a substance in a colloidal carrier is most commonly determined by the solubility characteristics of the drug as well as the polymer. Pharmaceutical compounds are usually soluble in either aqueous or non-aqueous solvents, which facilitates incorporation of these compounds into the nanoparticles by following the various emulsification techniques [3].
Furthermore, the inhibitory effect of ellagic acid on the proliferation of MCF-7 cells was ascribed to the initiation of cell cycle arrest [4].

Here we present a method for encapsulating EA into biodegradable poly(lactide-co-glycolide) (PLGA) nanoparticles using TWEEN ${ }^{\circledR} 20$ as a cosolvent and study its effect on the Human Breast Cancer, MCF7 cell line.

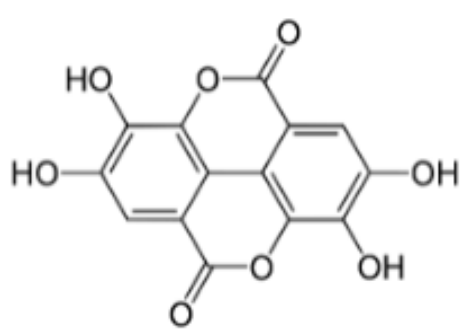

Fig. 1: Structure of ellagic acid

\section{MATERIALS AND METHODS}

\section{Materials}

Poly-D,L-lactic-co-glycolic acid (PLGA) with a copolymer ratio of D,Llactide to glycolide of 50:50(molecular weight 30,000-60,000) was purchased from Sigma-Aldrich (St Louis, MO, USA) and Ellagic acid was gifted by CSIR-North East Institute of Science and Technology, Jorhat. PVA was purchased from Loba Chemie Pvt Ltd (Mumbai, India). HPLC grade Acetone and Methanol was purchased from Spectrochem (Mumbai, India). All the other chemicals and solvents complied with the analytical grade and were purchased from Merck India. 


\section{Methodology}

\section{Preparation of nanoparticles and encapsulation}

Ellagic acid loaded PLGA nanoparticles were prepared using single emulsification (by probe sonication) and solvent evaporation with some modifications. Briefly, PLGA (35 mg) solution in acetone was mixed with EA(1 mg) solution in methanol and TWEEN ${ }^{\circledR} 20$ by bath sonication for 5 $\mathrm{min}$. The acquired emulsion was added to the aqueous solution $(15 \mathrm{ml})$ of varying amounts of $(0.1,0.3,0.5) \% \mathrm{w} / \mathrm{v}$ polyvinyl alcohol $(\mathrm{PVA})$ and sonicated for $10 \mathrm{~min}$ at $60 \mathrm{~W}$ amplitude using probe-sonicator (Hielscher, Germany). This final suspension was then magnetically stirred at $1000 \mathrm{rpm}$ overnight at room temperature for the removal of acetone. The resulted nanoparticles of EA were centrifuged in a cooling centrifuge at $11,000 \mathrm{rpm}$ for $10 \mathrm{~min}$ at $4{ }^{\circ} \mathrm{C}$, washed with deionized (Milli-Q) water to remove PVA and unencapsulated free EA, and lyophilized for $6 \mathrm{~h}$ to obtain a free-flowing powder [5].

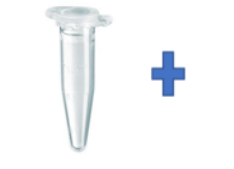

$25 \mathrm{mg}$ PLGA in

$1.7 \mathrm{~mL}$ acetone

$+0.2 \mathrm{~mL} \mathrm{MeOH}$

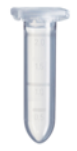

$1 \mathrm{mg} E A+0.6 \mathrm{~m}$ $\mathrm{MeOH}+20 \mu \mathrm{L}$ Tween 20

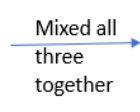
together
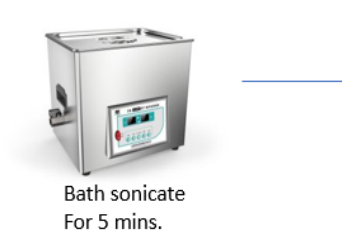

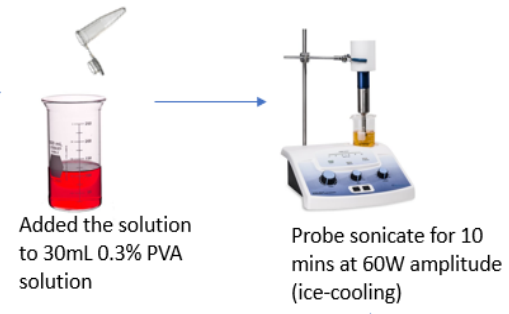

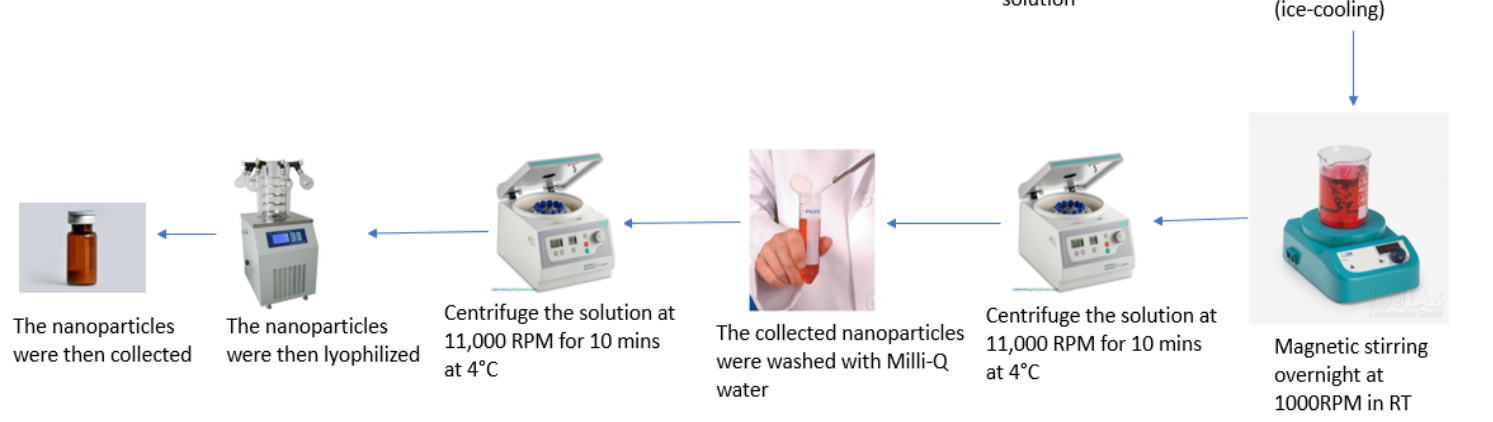

Fig. 2: Flow diagram of the EA loaded nano-encapsulation process

Table 1: Formulation and production for EA-NPs

\begin{tabular}{lll}
\hline Formulation code & Drug: polymer ratio & Stabilizer (\%) \\
\hline A & $1: 35$ & 0.1 \\
B & $1: 35$ & 0.3 \\
C & $1: 35$ & 0.5 \\
\hline
\end{tabular}

\section{Characterization}

Particle Size Distribution and Zeta Potential Analysis. The lyophilized EA-NPs were reconstituted with Milli-Q water prior to the measurements. The average particle size, size distribution, and polydispersity index (PDI) of the prepared nanoparticles were determined by Photon Correlation Spectroscopy using a Malvern NanoZS (Malvern Instruments, Worcestershire, United Kingdom). Zeta Potential was measured at $25^{\circ} \mathrm{C}$ using the same instrument. All the measurements were performed in triplicate; average values of three experiments were calculated and reported [6].

\section{Field emission-scanning electron microscopy (FE-SEM)}

The sampling for FE-SEM was done in two methods: (a) The dried nanoparticles was evaluated directly; (b) $20 \mu \mathrm{l}$ of re-dispersed solution of nanoparticles in Milli-Q water was pipetted out into a coverslip and air-dried. It was then placed in a dessicator for $24 \mathrm{~h}$ and then evaluated in a FESEM ZEISS, SIGMA (Carl Zeiss Microscopy, Germany) operating at $20 \mathrm{kV}$ accelerating voltage after proper placing on a carbon tube mounted on a SEM specimen stub coated with gold before observation [7]

\section{Evaluation}

\section{Drug loading and encapsulation efficiency of EA-NPs}

$10 \mathrm{mg}$ of the nano-formulation was weighed and then dissolved in 2 $\mathrm{ml}$ of Methanol and then sonicated in order to dissolve the PLGA nanoparticles completely. UV absorbance scans were taken of this solution obtained against methanol as blank at the specific $\lambda_{\max }$ of EA. Using the obtained value, the concentration of EA was calculated from the standard curve. Simultaneously, the Drug Loading,
Encapsulation Efficiency and Percentage Yield were calculated using the following formulae:

Drug loading contend with nanoparticles after their separation from the medium and to examine their drug content. It is calculated using the following equation [5]:

Drug loading content $(\%)=$ Weight of the drug in NPsWeight of the NPs x 100

Entrapment efficiency indicates about the \%drug that is successfully entrapped/adsorbed into nanoparticles. It is calculated as follows:

Encapsulation efficiency $(\%)=$ Weight of the drug in NPsInitial amount of drug x 100

\section{In vitro release}

Release of EA from the particles was determined by the dialysis membrane method. The nanoparticles were suspended in $1 \mathrm{ml} \mathrm{of} \mathrm{pH}$ 7.4 phosphate buffer and transferred to dialysis bag (molecular mass cut-off $12,000 \mathrm{D}$ ). The bag was placed in a $5 \mathrm{ml}$ glass vial containing $4 \mathrm{ml}$ of pH 7.4 phosphate buffer maintained at $37{ }^{\circ} \mathrm{C}$ and under magnetic stirring at $150 \mathrm{rpm}$. At fixed time intervals, the complete release medium was removed and replaced with $4 \mathrm{ml}$ of fresh medium. The amount of EA released into the medium was analyzed by UV spectrophotometry. The stability of EA in phosphate buffer $\mathrm{pH}$ 7.4 was also studied. Solutions of EA in pH 7.4 phosphate buffer $(6$ $\mathrm{mg} / \mathrm{ml}$ ) were prepared in triplicate, stored at $37^{\circ} \mathrm{C}$ and analyzed at predetermined time intervals for $15 \mathrm{~d}$ by UV spectrophotometry [3].

Note: Based on the above evaluation parameters an optimized formulation was selected for further processing and analysis.

\section{Application of drug release data on mathematical models}

Several mathematical equations which generally define the dissolution profile. Once an appropriate function has been selected, the evaluation of dissolution profile can be carried out and hence the drug release profile can be correlated with drug release kinetic models. Various mathematical models are employed to understand drug release kinetics which is explained below. 


\section{Zero order model}

According to the principles of pharmacokinetics, drug release from the dosage form can be represented by the equation:

$$
\begin{aligned}
& \mathrm{C}_{0}-\mathrm{C}_{\mathrm{t}}=\mathrm{K}_{0} \mathrm{t} \\
& \mathrm{C}_{\mathrm{t}}=\mathrm{C}_{0}+\mathrm{K}_{0} \mathrm{t}
\end{aligned}
$$

$\mathrm{C}_{\mathrm{t}}$ is the amount of drug released at time $\mathrm{t}$,

$\mathrm{C}_{0}$ is the initial concentration of drug at time $\mathrm{t}=0, \mathrm{~K}_{0}$ is the zero-order rate constant.

Thus, zero order kinetics defines the process of constant drug release from a drug delivery system and drug level in the blood remains constant throughout the delivery.

Hence to study the drug release kinetics data obtained from in vitro dissolution study is plotted against time i.e., cumulative drug release vs. time.

Hence the slope of the above plot gives the zero-order rate constant and the correlation coefficient of the above plot will give the information whether the drug release follows zero order kinetics or not [8].

\section{First order model}

The release of drug which follows first order kinetics can be represented by the equation:

$$
\mathrm{DC} / \mathrm{dt}=-\mathrm{K}_{1} \mathrm{C}
$$

$\mathrm{K}_{1}$ is the first order rate constant, expressed in time-1 or per hour.

Hence it can be defined as that first order process is the one whose rate is directly proportional to the concentration of drug undergoing reaction i.e., greater the concentration faster the reaction. Hence, it follows linear kinetics.

After rearranging and integrating the equation,

$$
\log \mathrm{C}=\log \mathrm{C}_{0}-\mathrm{K}_{1} \mathrm{t} / 2.303
$$

$\mathrm{K}_{1}$ is the first order rate equation expressed in time- 1 or per hour,

$\mathrm{C}_{0}$ is the initial concentration of the drug,

$\mathrm{C}$ is the percent of drug remaining at time $t$.

Hence to study the drug release kinetics data obtained from in vitro dissolution study is plotted against time i.e., $\log \%$ of drug remaining vs. time and the slope of the plot gives the first order rate constant.

The correlation coefficient of the above plot will give the information whether the drug release follows first order kinetics or not [8].

\section{Higuchi model}

The release of a drug from a drug delivery system (DDS) involves both dissolution and diffusion. Several mathematical equations models describe drug dissolution and/or release from DDS.

In the modern era of controlled-release oral formulations, 'Higuchi equation' has become a prominent kinetic equation in its own right, as evidenced by employing drug dissolution studies that are recognized as an important element in drug delivery development. Today the Higuchi equation is considered one of the widely used and the most well-known controlled-release equation.

The classical basic Higuchi equation is represented by

$$
\mathrm{Q}=\mathrm{AD}(2 \mathrm{C} 0-\mathrm{Cs}) \mathrm{Cst}
$$

Where $\mathrm{Q}$ is the cumulative amount of drug released in time t per

Unit area, C0 is the initial drug concentration, Cs is the drug solubility

In the matrix and $\mathrm{D}$ is the diffusion coefficient of the drug molecule in the matrix.

This relation is valid until total depletion of the drug in the dosage form is achieved. To study the dissolution from a planar heterogeneous matrix system, where the drug concentration in the matrix is lower than its solubility and the release occurs through porous system, the expression can be given by equation:

$$
\mathrm{Q}=(\mathrm{D} \delta / \tau)(2 \mathrm{C}-\delta \mathrm{Cs}) \mathrm{Cst}
$$

Where $\mathrm{D}$ is the diffusion coefficient of the drug molecule in the solvent; $\delta$ is the porosity of the matrix; $\tau$ is the tortuisity of the matrix and $\mathrm{Q}, \mathrm{A}, \mathrm{Cs}$ and $\mathrm{t}$ have the meaning described above.

Tortuisity is defined as the dimensions of radius and branching of the pores and canals in the matrix. After simplifying the above

equation, Higuchi equation can be represented in the simplified form

$$
\mathrm{Q}=\mathrm{K}_{\mathrm{H}} \times \mathrm{t}^{1 / 2}
$$

Where, $\mathrm{K}_{\mathrm{H}}$ is the Higuchi dissolution constant.

The data obtained were plotted as cumulative percentage drug release versus square root of time. Therefore, the simple Higuchi model will result a linear $\mathrm{Q}$ versus $\mathrm{t}^{1 / 2}$ plot having gradient, or slope, equal to $\mathrm{K}_{\mathrm{H}}$ and we say the matrix follows $\mathrm{t}^{1 / 2}$ kinetics.

Hence if the correlation coefficient is higher for the above plot then we can interpret that the prime mechanism of drug release is diffusion-controlled release mechanism.

It is important to note that a few assumptions are made in this Higuchi model. These assumptions are:

(i) The initial drug concentration in the system is much higher than the matrix solubility

(ii) Perfect sink conditions are maintained

(iii) The diffusivity of the drug is constant and

(iv) The swelling of the polymer is negligible. The sink conditions are achieved by ensuring the concentration of the released drug in the release medium never reaches more than 10 per cent of its saturation solubility [8].

\section{Korsmeyer-peppas model}

Once it has been ascertained that the prime mechanism of drug release is diffusion controlled from Higuchi plot then it comes the release of drug follows which type of diffusion. To understand the dissolution mechanisms from the matrix, the release data were fitted using the well-known empirical equation proposed by Korsmeyer and Peppas. Korsmeyer and Peppas put forth a simple relationship which described the drug release from a polymeric system follow which type of dissolution and he represented an equation as:

$\mathrm{Mt} / \mathrm{M} \infty=\mathrm{Kkptn}$

$\mathrm{Mt} / \mathrm{M} \infty$ is a fraction of drug released at time $\mathrm{t}$,

$\log (M t / M \infty)=\log K_{k p}+n \log t$,

Mt is the amount of drug released in time $t$,

$\mathrm{M} \infty$ is the amount of drug released after time $\infty, \mathrm{n}$ is the diffusional exponent or drug release exponent, $\mathrm{K}_{\mathrm{kp}}$ is the Korsmeyer release rate constant.

To study release kinetics a graph is plotted between log cumulative $\%$ drug release $\log (\mathrm{Mt} / \mathrm{M} \infty)$ vs. $\log$ time $(\log \mathrm{t})[8]$.

The aforementioned models were applied on the release profile of the formulated EA-NPs and the evaluation was done in graphical representation.

\section{Long-term stability study}

EA-NPs prepared by single emulsification and solvent evaporation technique were stored at $4 \pm 2{ }^{\circ} \mathrm{C}$ for $3 \mathrm{mo}$. After storage for $3 \mathrm{mo}$ samples were analysed to determine particle size and zeta potential value as per methods mentioned previously. Experiments were done in triplicate.

\section{Cell culture}

Human breast cancer cell line, MCF-7, derived from human Caucasian breast adenocarcinoma represents the advanced stages of this disease and was purchased from National Centre for Cell Sciences, Pune. 
Cell growth was performed in DMEM medium with $10 \%(\mathrm{v} / \mathrm{v})$ heatinactivated fetal bovine serum (FBS) and $2 \mathrm{mmol}$ L-glutamine, supplemented with $100 \mathrm{U} / \mathrm{ml}$ Penicillin, $100 \mu \mathrm{g} / \mathrm{ml}$ Streptomycin and with $5 \mu \mathrm{g} / \mathrm{ml}$ Kanamycin. Cells were maintained in a $95 \%$ humidified incubator with $5 \% \mathrm{CO} 2$ at $37{ }^{\circ} \mathrm{C}$, and were passaged with trypsinization every fourth day. The stock solution was prepared in dimethyl sulfoxide (DMSO) and stored-20 ${ }^{\circ} \mathrm{C}$ until use. The aliquots used in this study were freshly prepared for each experiment with a final DMSO concentration of $0.1 \%$ [4].

\section{In vitro cytotoxicity studies}

The effect of free EA and EA-NPs on the viability of MCF-7 cell line was determined by MTT [3-(4,5-dimethylthiazol-2-yl)-2,5diphenyltetrazolium bromide] assay. The MCF-7, human breast cancer cells were seeded in 96 well plates containing growth medium $(1 \times 104$ cells per well) and incubated (for 24 and $48 \mathrm{~h})$ at $37{ }^{\circ} \mathrm{C}$ in humidified air containing $5 \%$ CO2. The cells were exposed to blank nanoparticles, free EA, and EA-NPs containing EA in different concentrations $(5,25,50,75,100 \mu \mathrm{M})$. After 24 and $48 \mathrm{~h}$ of incubation, media containing the drug was removed. Then $10 \mu \mathrm{l}$ of MTT solution ( $5 \mathrm{mg} / \mathrm{ml}$ in PBS) and $90 \mu \mathrm{l}$ of medium were added to each well, incubated for another $4 \mathrm{~h}$ before the addition of dimethylsulfoxide $(150 \mu \mathrm{l})$, and the optical density (OD) at $540 \mathrm{~nm}$ was measured using a microplate reader (GENios, Austria). Cytotoxicity was expressed in terms of IC50 calculated from the cell viability data representing the drug concentration in which cell growth was inhibited by $50 \%$ [9].

\section{RESULTS AND DISCUSSION}

\section{Preparation of drug loaded nanoparticles}

In recent years various attempts have been made by many researchers in the development of novel drug delivery systems using nanoparticles to minimize toxicity, increase stability and specificity, increase bioavailability, and achieve sustained release of different hydrophilic and hydrophobic drugs. Depending on the physicochemical nature of the polymer and the drug to be loaded, several techniques have been adopted for the preparation of nanoparticles. Among them, the emulsion solvent evaporation method is most frequently used for hydrophobic drugs. In this study, The EA loaded PLGA nanoparticles were prepared by adopting a modified single emulsification (by sonication) and solvent evaporation technique where TWEEN ${ }^{\circledR} 20$ was employed as cosolvent. The solution of EA and PLGA in acetone was sonicated for emulsification into an aqueous phase containing PVA as surfactant. If performed under cold condition, sonication induces an increase in temperature and restores the integrity of drug molecule. After solvent evaporation, the precipitated nanoparticles were washed several times with distilled water to remove the surfactant and unencapsulated free drug.

\section{Characterization}

Table 1 shows the mean particle size, polydispersity index, zeta potential, encapsulation efficiency and drug loading of the 3 batches of EA-NPs prepared having formulation code A, B and C. Assessing the particle size, zeta potential and PDI it was found that the formulation B was the most optimized due to the smaller particle size, high encapsulation efficiency, drug loading and low PDI values.

Table 2 shows the mean particle size, yield, encapsulation efficiency, drug loading and zeta potential of optimized EA-NPs. The average size of the particles as determined by DLS experiment was $174 \pm 2.50$ $\mathrm{nm}$ when prepared by single emulsion solvent evaporation (fig. 3) which is in the acceptable nanoparticle range. Since, nanoparticles size in the range of $10-200 \mathrm{~nm}$ are ideal for tumour accumulation. Hence, the obtained PLGA nanoparticles are ideal for tumourspecific drug delivery.

Nanoparticles exhibited narrow size distribution with polydispersity index (PDI) of 0.011 when prepared by single emulsification technique (fig. 3).

Zeta potential is an index of stability of nanoparticles. Higher the magnitude, irrespective of charge type, higher the stability and monodispersity expected. PVA alone resulted in anionic particles. The zeta potential value of nanoparticles was approximately $-23.8 \mathrm{mV}$ (pH 5.50) when they were prepared by single emulsification method (fig. 4).

Single emulsification technique produced smooth spherical EA-NPs of comparatively small size with relatively narrow size distribution; these were used for further characterization and evaluation.

The encapsulation efficiencies achieved with this method are very good considering the poor solubility profile of EA. PVA alone provided a good encapsulation efficiency of about $44.8 \%$.

The EA-NPs formulation was stored at $4 \pm 2{ }^{\circ} \mathrm{C}$ for 3 mo and thereafter the particle size, zeta potential and PDI were evaluated.

The particle size of nanoparticles slightly increased to $176 \mathrm{~nm}$ and subsequent rise in polydispersity index. This could be due to the formation of aggregates during storage but change was not very significant. The zeta potential of the EA-NPs did not change significantly during storage.

\section{Size (d.n... $\quad \%$ Intensity: St $\operatorname{Dev}$ (d.n...}

Z-Average (d.nm): 174.2

Peak 1: $\quad 180.3$

$100.0 \quad 37.76$

Pdl: 0.011

Peak 2: $\quad 0.000$

0.0

0.000

Intercept: 0.962

Peak 3: $\quad 0.000$

0.0

0.000

Result quality Refer to quality report

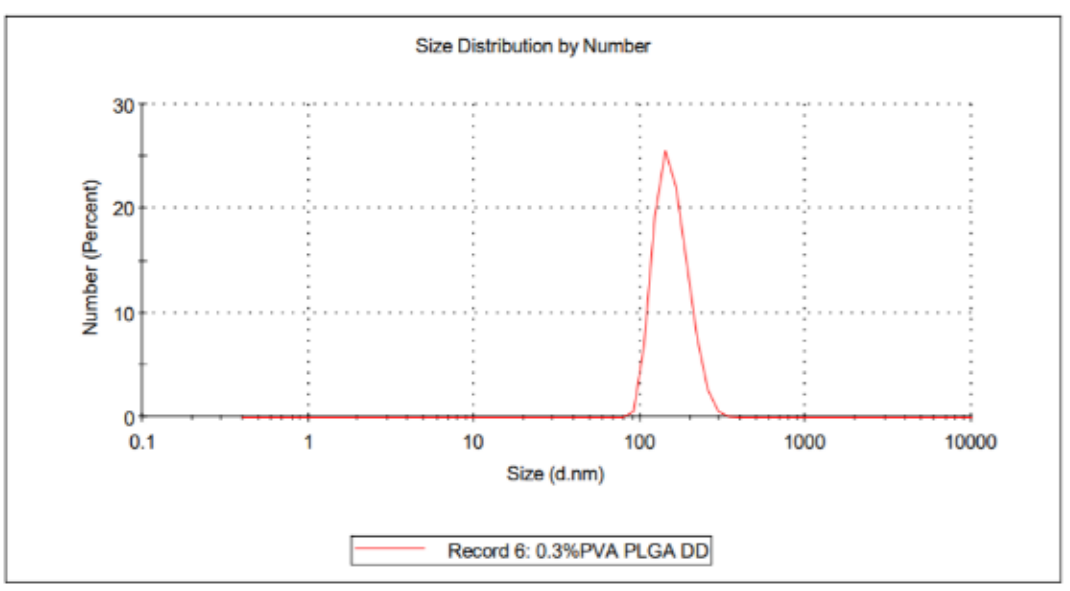

Fig. 3: The average diameter and size distribution of nanoparticles measured by zeta sizer 
Table 1: Particle size, PDI, zeta-potential, encapsulation efficiency and drug loading of prepared nanoparticles

\begin{tabular}{llllll}
\hline Formulation code & Particle size (nm) & Zeta potential (mV) & PDI & Encapsulation efficiency (\%) & Drug loading (\%) \\
\hline A & $196 \pm 10.23$ & $-20.5 \pm 5.38$ & 0.134 & $40 \pm 6.45$ & $3.2 \pm 2.50$ \\
B & $174 \pm 2.50$ & $-23.8 \pm 5.00$ & 0.011 & $44 \pm 8.42$ & $4 \pm 1.92$ \\
C & $210 \pm 27.82$ & $-19.07 \pm 5.29$ & 0.126 & $43 \pm 7.90$ & $3.89 \pm 1.58$ \\
\hline
\end{tabular}

Table 2: Size, encapsulation efficiency, yield and the physicochemical characteristics of the optimized formulation $(n=3)$

\begin{tabular}{ll}
\hline Encapsulation efficiency & $44 \pm 8.42$ \\
Drug loading & $4 \pm 1.92$ \\
Yield (\%) & $50 \pm 4.95$ \\
Size (nm) & $174 \pm 2.50$ \\
PDI & 0.011 \\
Zeta potential (mV) & -23.8 \\
\hline
\end{tabular}

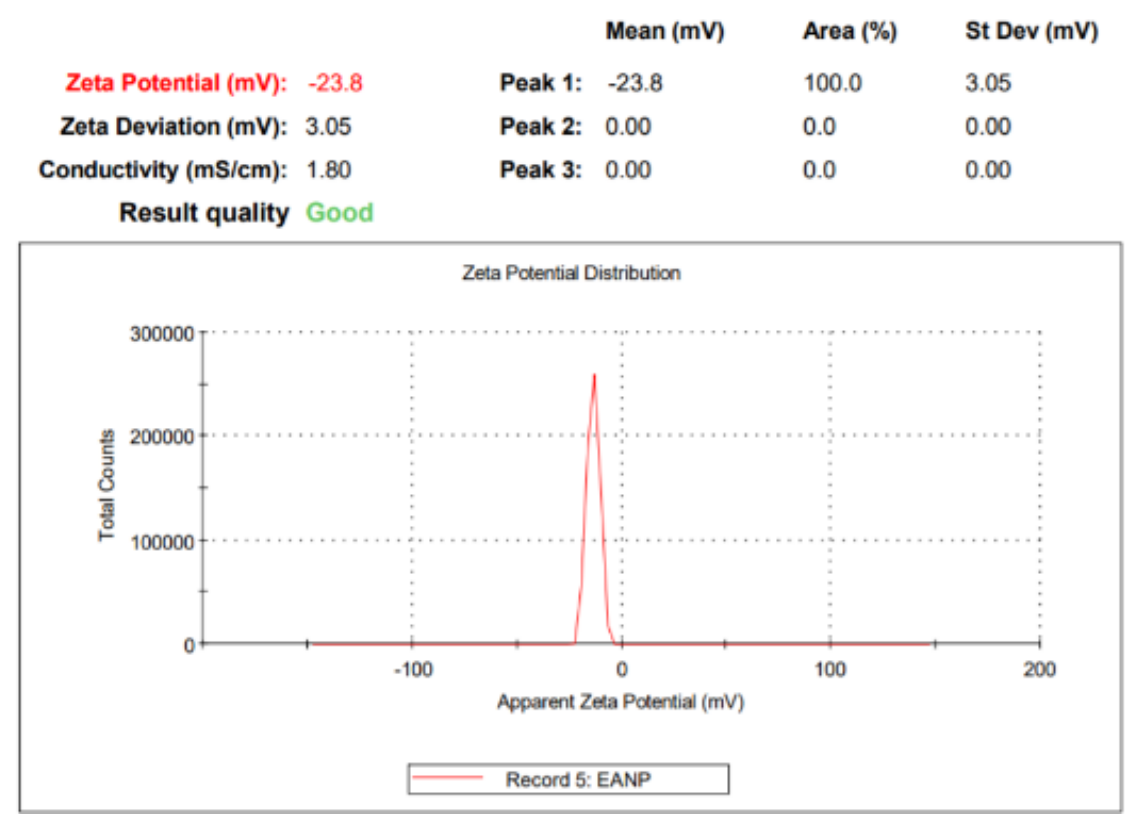

Fig. 4: Zeta potential of nanoparticles measured by zeta sizer

Field emission scanning electron microscopy (FE-SEM) characterization of nanoparticles

Microphotographs of EA loaded PLGA nanoparticles was obtained by scanning electron microscopy (Zeiss). Fig. 5 shows the Scanning Electron Microscope (SEM) image of the EA loaded PLGA nanoparticles. It confirmed that the NPs have smooth and spherical shape. The resulting NPs were predominantly spherical and of uniform size and shape.

\section{Evaluation}

\section{In vitro release}

The cumulative release of EA from nanoparticles prepared using PVA is shown in Graph I. The initial release is rather rapid, followed by a slower sustained phase. The particles prepared using PVA as stabilizer showed very fast release, about $50 \%$ of drug being released over a period of $5 \mathrm{~d}$. The release rate of drug from nanoparticles can be affected by various parameters/properties like physicochemical properties of drug and polymer, solvents/stabilizers used to prepare nanoparticles and most importantly, the size of the particles. Presence of hydrophilic groups of PVA allows better penetration of water molecules into the polymer matrix, which enables faster release. Degradation of EA in phosphate buffer was also observed with time. It is evident from Graph I that $70 \%$ of the drug has been degraded by day 10 , while release of the drug from the nanoparticles beyond day 10 suggests the protective effect of the polymer preventing degradation of drug. Around $71.98 \%$ of EA was released from nanoencapsulation over the period of $15 \mathrm{~d}$, thus providing a controlled and sustained release pattern.

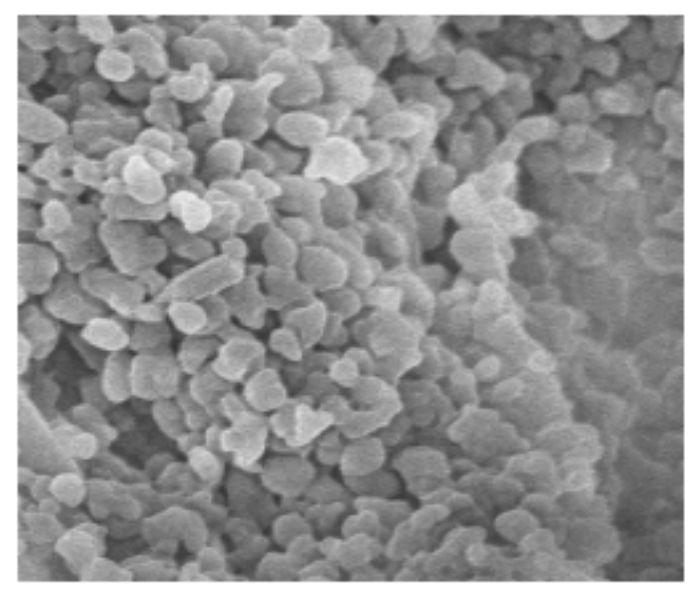

Fig. 5: SEM image of EA-NPs prepared by single emulsification and solvent evaporation technique 


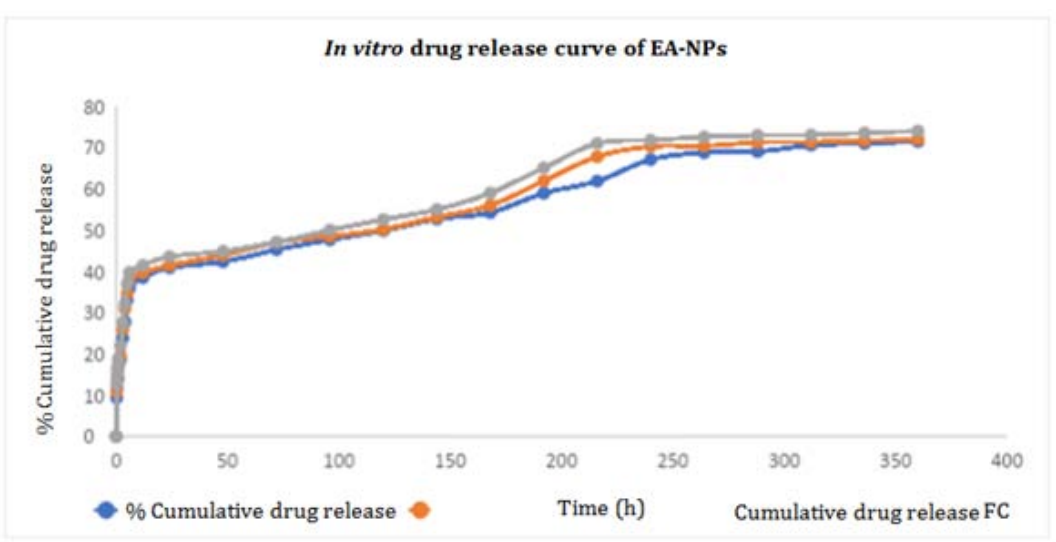

Graph 1: Release profile of EA from PLGA nanoparticles in pH 7.4 phosphate buffer at $37^{\circ} \mathrm{C}(\mathrm{n}=3)$

\section{Mathematical models for drug release}

In order to determine drug release pattern of the EA loaded NPs, the release data of the optimized formulation (FB) were substituted to Zero, First, Higuchi and Korsemeyer Peppas model. The $\mathrm{R}^{2}$ value and rate constants/release exponent values determined from the data of drug release following different kinetic models are given in table 2 . More linearity (by assessing $\mathrm{R}^{2}$ values) was detected in KorsmeyerPeppas plot $\left(\mathrm{R}^{2}=0.9341\right)$ followed by Higuchi's $(\mathrm{R} 2=0.9118)$. This describes the release of drug from a polymeric system. The formulation also tends to have more linearity towards first order release $\left(R^{2}=0.9077\right)$ than the zero-order release $\left(R^{2}=0.8051\right)$

\section{In vitro cytotoxicity assay}

EA-NPs, free EA, and drug free PLGA NPs were evaluated by assessing cell viability using MTT assay on human breast cancer, MCF-7 cell line. The study was conducted using different concentrations (ranging between 5 and $100 \mu \mathrm{M}$ ) of drug loaded nanoparticles containing EA. Two sets of experiments were done for 24 and $48 \mathrm{~h}$ durations. The effect was more pronounced after $48 \mathrm{~h}$ incubation. Cytotoxicity was expressed in terms of \%cell viability against concentration. Both free $\mathrm{EA}$ and EA-NPs displayed concentration dependent cytotoxicity (Graph VI). The IC $\mathrm{I}_{50}$ values of free EA and EA-NPs were 29 and $9 \mu \mathrm{M}$ respectively following $48 \mathrm{~h}$ incubation with the cell line $(\mathrm{n}=3)$. EANPs exhibited dose-dependent activity in comparison to free drug. A higher concentration of EA may be delivered to the intracellular space due to small size of the nanoparticle. Sustained release of the drug from inner polymer matrix of nanoparticulated formulation resulted pronounced cytotoxic effect [10]. Better efficacy and lower $\mathrm{IC}_{50}$ were observed for EA-NPs whereas no cellular cytotoxic effects were observed when drug free PLGA NPs were exposed to cell line; this confirms the safe nature of the copolymer.

Table 2: Data of drug release kinetics of experimental nanoparticles

\begin{tabular}{ll}
\hline Release media & Phosphate buffer saline $(\mathbf{p H}=\mathbf{7 . 4})$ \\
\hline Zero order & $\mathrm{y}=0.1597 \mathrm{x}+26.061$ \\
& $\mathrm{R}^{2}=0.8051$ \\
& $\mathrm{~K}_{0}=0.1597$ \\
First order & $\mathrm{y}=-0.0014 \mathrm{x}+1.8694$ \\
& $\mathrm{R}^{2}=0.9077$ \\
Higuchi kinetics & $\mathrm{K}_{1}=-0.0014$ \\
& $\mathrm{y}=3.1315 \mathrm{x}+18.118$ \\
& $\mathrm{R}^{2}=0.9118$ \\
Korsemeyer peppas & $\mathrm{K}_{\mathrm{H}}=3.1315$ \\
kinetics & $\mathrm{y}=0.2095 \mathrm{x}+1.3176$ \\
& $\mathrm{R}^{2}=0.9341$ \\
& $\mathrm{n}=0.2095$ \\
\hline
\end{tabular}

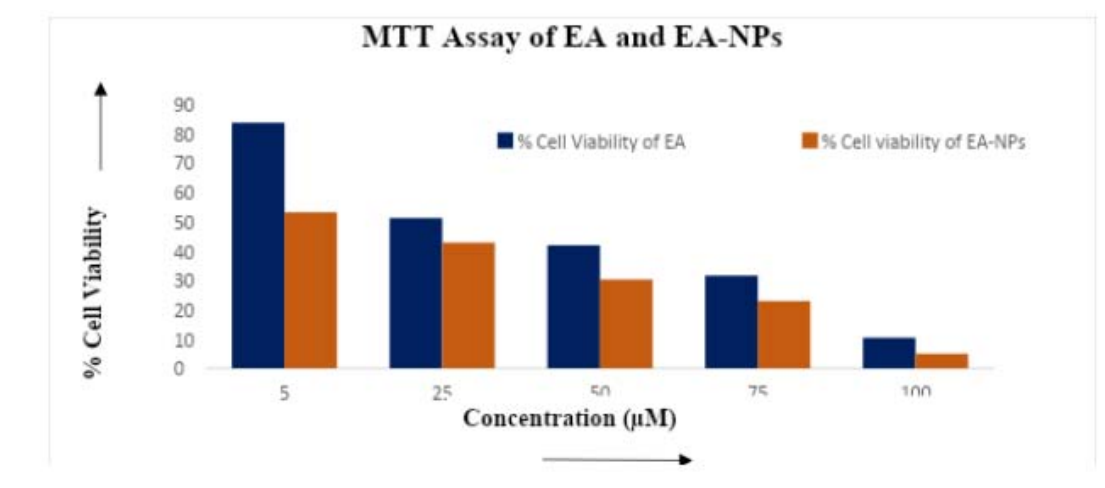

Graph 2: MTT assay of EA and EA-NPs on MCF-7 cells at different concentrations for $48 \mathrm{~h}$

\section{CONCLUSION}

Nanoparticulated drug delivery systems have recently attracted considerable attraction for targeted drug delivery of various anticancer drugs ranging from synthetic compounds to natural products. However, the evaluation of their biological performance is still highly challenging. Anticancer potentials of EA have been reported by various workers in this field. We reported an efficient EA delivery system using the single emulsification (by probe sonication) and solvent evaporation incorporating the biocompatible polymer PLGA as the drug carrier using TWEEN ${ }^{\circledR} 20$ as the co-solvent. The optimized formulation was found to be having an average particle size of 174.2 $\mathrm{nm}$, which is ideal for tumour accumulation. Nanoparticles exhibited narrow size distribution with polydispersity index (PDI) of 0.011 when prepared by single emulsification technique and thereby indicate uniform monodispersity of the formulation. The zeta potential value of nanoparticles was approximately $-23.8 \mathrm{mV}$ (pH 5.50). Hence indicating a stable formulation. The encapsulation efficiencies achieved with this method are very good considering the poor solubility profile of EA. PVA alone provided a good encapsulation 
efficiency of about $44.8 \%$. The SEM images confirmed that the NPs have spherical shape. The resulting NPs were predominantly spherical and of uniform size and shape. The in vitro drug release study revealed slow and sustained release of the drug which could be exploited for potential therapy. Kinetic release modelling was done using the in vitro release data and the release was found to be most linear to Korsemeyer Peppas release which indicates the release of a drug from a polymeric system. MTT assay revealed the pronounced cytotoxic effect of the formulation. All these proved the clinical significance of the EA loaded nanoparticulated drug delivery system.

\section{ACKNOWLEDGEMENT}

The authors wholeheartedly acknowledge the laboratory facilities and guidance provided by. The Director of Regional Institute of Paramedical and Nursing Sciences, Aizawl, India for carrying out this work and giving such a great opportunity to explore the recent technology and development in the field of Pharmaceutical Sciences.

\section{FUNDING}

Nil

\section{AUTHORS CONTRIBUTIONS}

All the authors have contributed equally.

\section{CONFLICT OF INTERESTS}

Declared none

\section{REFERENCES}

1. Garcia Diaz M, Foged C, Nielsen HM. Improved insulin loading in poly (lactic-co-glycolic) acid (PLGA) nanoparticles upon selfassembly with lipids. Int J Pharm 2015;482:84-91.
2. Das PJ, Paul P, Mukherjee B, Mazumder B, Mondal L. Pulmonary delivery of voriconazole loaded nanoparticles providing a prolonged drug level in lungs: a promise for treating fungal infection. Mol Pharm 2015;12:2651-64.

3. Bala I, Bhardwaj V, Hariharan S, Kharade SV, Roy N. Sustained release nanoparticulate formulation containing antioxidantellagic acid as potential prophylaxis system for oral administration. J Drug Target 2006;14:27-34.

4. Zhang T, Chen HS, Wang LF, Bai MH, Wang YC. Ellagic acid exerts anti-proliferation effects via modulation of Tgf-B/Smad3 signaling in MCF-7 breast cancer cells. Asian Pac J Cancer Prev 2014;15:273-6.

5. Baishya R, Nayak DK, Kumar D, Sinha S, Gupta A. Ursolic acid loaded PLGA nanoparticles: in vitro and in vivo evaluation to explore tumor targeting ability on B16F10 melanoma cell lines. Pharm Res 2016;33:2691-703.

6. Dang S, Sharma D, Philip G, Gabrani R, Ali J. Dual agents loaded polymeric nanoparticle: effect of process variables. Int J Pharm Investig 2015;5:155.

7. Babos G, Biro E, Meiczinger M, Feczko T. Dual drug delivery of sorafenib and doxorubicin from PLGA and PEG-PLGA polymeric nanoparticles. Polymers 2018;10:1-12.

8. Baishya H. Application of mathematical models in drug release kinetics of carbidopa and levodopa er tablets. J Dev Drugs 2017;6:1-8.

9. Kim HA, Lee RA, Moon BI, Choe KJ. Ellagic acid shows different anti-proliferative effects between the MDA-MB-231 and MCF-7 human breast cancer cell lines. J Breast Cancer 2009;12:85-91.

10. Kamaly N, Xiao Z, Valencia PM, Radovic Moreno AF, Farokhzad OC. Targeted polymeric therapeutic nanoparticles: design, development and clinical translation. Chem Soc Rev 2012;41:2971-3010. 